\title{
Design and implementation of the inverted pendulum optimal controller based on hybrid genetic algorithm
}

\author{
Bingbing Wu, Chaoying Liu, Xueling Song, Xuesong Wang \\ Institute of Electrical Engineering,Hebei University of Science and Technology, Shijiazhuang, Hebei, \\ 050000, China
}

Keywords: Double inverted pendulum; LQR; Genetic Algorithms

\begin{abstract}
The inverted pendulum is a multivariable, rapid, nonlinear unstable system. LQR optimal control is often used in inverted pendulum control for its good stability. According to optimal determination problem of the weighting matrix $\mathrm{Q}$ and $\mathrm{R}$ in $\mathrm{LQR}$, an improved hybrid genetic algorithm is adopted in LQR optimal controller design in double inverted pendulum and the optimal parameter values can be find by effective use of its intelligent search, progressive optimization features characters. This controller is successfully used in double inverted pendulum. The experimental results show that the optimal controller designed by this method has small overshoot and fast response. It can actually control the inverted pendulum system to achieve stability and proves the feasibility of the control method.
\end{abstract}

\section{Introduction}

Inverted pendulum equipment is recognized as the typical experiment device of automatic control theory. It has the characteristics of absolute instability, high order time, multi-variable and strong coupling. The inverted pendulum can be well used to testing various control theories and methods, thus attracted the attention of many researchers and scholars. The control method has a wide range of uses in the field of military industry, aerospace science and technology, robot and general industrial process. In recent years, many scholars have used the classical control theory, modern control theory and a variety of intelligent control methods [1,2]in this system and successfully achieved stability control of inverted pendulum system.

Due to the special architecture of double inverted pendulum system, the traditional method of state feedback control is difficult to obtain satisfactory results. But the linear quadratic optimal design methodhas the advantages of good robustness in the double inverted pendulum system, so it has been widely used in the design of control system[3] .

A key problem of LQR controller design is the adjustment of matrix $Q$ and R. However, quadratic weighting matrix $\mathrm{Q}$ and $\mathrm{R}$ are chosen by trial and error method in most of the literatures. There is no rule to follow for $\mathrm{Q}$ and $\mathrm{R}$ option and it usually depends on the designer's experience. Complex trial and error not only affects the design efficiency, but also can not guarantee the system meets specific performance indicators.

To solve these problems, the article uses improved genetic algorithm optimization design [4-6] to find the optimal linear quadratic control weighting matrix Q and $\mathrm{R}$ for the double inverted pendulum system.

\section{Double inverted pendulum dynamic model}

Double inverted pendulum structure is mainly composed of a horizontal rail, a car and double pendulum and it connected by bearing between the car and pendulum. Each bearing joints fixed potential, they are used to detect the double pendulum plumb line offset angle. Relative orbit the center of the car' s position is also offset by the same fixed pulley shaft potentiometers testing. The car can move freely on the horizontal guide rail by the control of the motor, belt pulley, a transmission, the two swing rod can rotate freely in a vertical plane. This paper takes GLIP2002 linear double inverted pendulum[7] as the model. Ignoring resistance and all kinds of 
friction, the inverted pendulum system is abstracted the car and homogeneous rod system, and it is shown in figure 1.

System specific parameters are as follows: the car quality $\mathrm{M}$ is $1.096 \mathrm{~kg}$, the pendulum one quality M1 is $\quad 0.05 \mathrm{~kg}$, pendulum two quality M2 is $0.13 \mathrm{~kg}$, goniometer potentiometer quality M3 is $0.236 \mathrm{~kg}$, the center of rotation of the pendulum rod centroid distance to $\mathrm{L} 1$ is $0.0775 \mathrm{~m}$, the distance $\mathrm{L} 2$ from rotation center of the pendulum to its centroid is $0.25 \mathrm{~m}$, The acceleration of gravity $g$ is $9.8 \mathrm{~m} / \mathrm{s}^{2}$. Wherein $\theta_{1}$ is the pendulum 1 angle in the vertical direction ; $\theta_{2}$ is pendulum 2 angel in vertical direction; $\mathrm{F}$ is the external force acting on the system; $x$ is the displacement of the car.

Inverted pendulum system control goal is when exerting force $\mathrm{F}$ to the car, ensuring the swinging rod upright and making the car stay in the specified location.Let $\mathrm{X}=\left[x, \theta_{1}, \theta_{2}, \dot{x}, \dot{\theta}_{1}, \dot{\theta}_{2}\right]^{T}$, $\mathrm{Y}=\left[x, \theta_{1}, \theta_{2}\right]^{T}$. Among them, $x$ and $\dot{x}$ represent trolley displacement and velocity, $\theta_{1}, \theta_{2}, \dot{\theta}_{1}, \dot{\theta}_{2}$ represent separately the swing rod one and swing rod two angle and the angular velocity. After linearization and substituting the parameters, the system's state space equation can be got.

$$
\begin{aligned}
& {\left[\begin{array}{c}
\dot{x} \\
\dot{\theta}_{1} \\
\dot{\theta}_{2} \\
\ddot{x} \\
\ddot{\theta}_{1} \\
\ddot{\theta}_{2}
\end{array}\right]=\left[\begin{array}{cccccc}
0 & 0 & 0 & 1 & 0 & 0 \\
0 & 0 & 0 & 0 & 1 & 0 \\
0 & 0 & 0 & 0 & 0 & 1 \\
0 & 0 & 0 & 0 & 0 & 0 \\
0 & 86.69 & -21.62 & 0 & 0 & 0 \\
0 & -40.31 & 39.45 & 0 & 0 & 0
\end{array}\right]\left[\begin{array}{c}
x \\
\theta_{1} \\
\theta_{2} \\
\dot{x} \\
\dot{\theta}_{1} \\
\dot{\theta}_{2}
\end{array}\right]+\left[\begin{array}{c}
0 \\
0 \\
0 \\
1 \\
6.64 \\
-0.088
\end{array}\right] u} \\
& Y=\left[\begin{array}{c}
x \\
\theta_{1} \\
\theta_{2}
\end{array}\right]=\left[\begin{array}{cccccc}
1 & 0 & 0 & 0 & 0 & 0 \\
0 & 1 & 0 & 0 & 0 & 0 \\
0 & 0 & 1 & 0 & 0 & 0
\end{array}\right]\left[\begin{array}{c}
x \\
\theta_{1} \\
\theta_{2} \\
\ddot{x} \\
\dot{\theta}_{1} \\
\dot{\theta}_{2}
\end{array}\right]+\left[\begin{array}{c}
0 \\
0 \\
0
\end{array}\right] u
\end{aligned}
$$

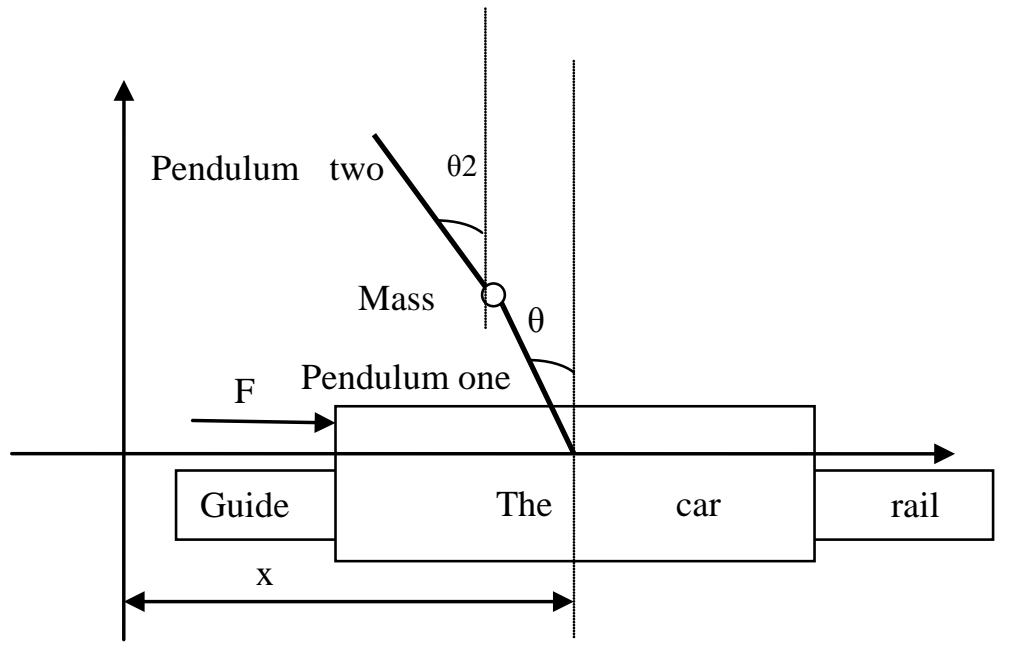

Fig .1: The line double inverted pendulum physical model

Using MATLAB to calculate the state control matrix and output controllability matrix rank, it can be concluded that the system state and output are controlled, and the system is observable. 


\section{LQR controller and improved genetic algorithm}

Linear quadratic controller.Linear quadratic optimal state regulator theory in modern control theory is the earliest development and the most mature state space design method. In practical engineering applications they are very common and widespread. It is one of the most important achievements of modern control theory.

Linear quadratic equation refers to the state of the system is linear, performance index functions are quadratic of the state variables and control variables. To seek optimal control law $\mathrm{u}(\mathrm{t})$, it needs to transfer the system from the initial state to near zero, and get a minimum value of the performance index.

General linear quadratic problem can be expressed as follows. Set up the linear model of controlled object:

$$
\left\{\begin{array}{l}
\dot{X}(t)=A X(t)+B u(t) \\
Y(t)=C X(t)+D u(t)
\end{array}\right.
$$

Among them: $X(t)$ is $n$ dimensional state vector; $u(t)$ is $m$ dimensional control vector; $Y(t)$ is $\boldsymbol{I}$ dimensional output vector. A, B, C, D are the appropriate constant matrix. Assuming vector $u(t)$ is unconstrained. Now introduce linear quadratic performance index function, as shown in the following formula:

$$
J=\frac{1}{2} X^{T}(t) S X(t)+\frac{1}{2} \int_{t_{0}}^{t_{f}}\left[X^{T}(t) Q(t) X(t)+u^{T}(t) R(t) u(t)\right] d t
$$

Among them: $Q$ is $6 \times 6$ dimensional symmetric positive semi-definite matrix, $R$ is $1 \times 1$ dimensional positive definite real symmetric matrix, and termination time for control action is $t_{f}$. To the constant coefficient optimal quadratic regulator problems, if making the minimum optimal control $J$ exist and unique, the necessary and sufficient condition is:

$$
u(t)=-k * X(t)=R^{-1} B^{T} P X(t)
$$

Where, $K$ is the final optimal state feedback control matrix, $P$ is the following algebra Riccati Symmetric positive definite solution of the equation:

$$
P(t) A+A^{T} P(t)-P(t) B R^{-1} B^{T} P(t)+Q=0
$$

The solution of the $P$ in Riccatican be obtained after constant feedback gain matrix:

$$
K=R^{-1} B^{T} P
$$

It can be seen, $P$ matrix depends on $A, B, Q$ and $R$ matrix, and $A, B$ are determined by the structure and parameters of the system respectively, so $P$ really depends on the weight matrix $Q$ and $R$.

In this paper, taking into account the actual situation of double inverted pendulum system in running, we should use the infinite time state regulator control system. When the time $t_{f}$ tends to infinity, the final system of state deviation is zero. The evaluation index system for the performance can be simplified as:

$$
J=\frac{1}{2} \int_{0}^{\infty}\left[X^{T}(t) Q(t) X(t)+u^{T}(t) R(t) u(t)\right] d t
$$

Improved Genetic Algorithm Design. Genetic algorithm is a kind of adaptive global optimization probability search algorithm and simulation for the formation of biological genetic in nature and evolutionary process. The basic idea is: the solution of the optimization problem is searched from a representative initial group, this set of solutions called population. The population consists of a number of genes coding[9] for individual components, and each individual is called chromosomes. Through the different chromosome copy it crossover or mutation to generate new individuals. According to the principle of survival of the fittest, the selection and elimination of the problem domain of individuals bases on individual fitness in size in each generation. Each generation evolve and compute the optimal approximate solution. 
Compared with the traditional (enumeration, heuristic, etc.) genetic algorithm, optimization method takes biological evolution as the prototype, has very good convergence. It has the advantages of less computing time, higher robustness in the calculating accuracy. But now, the work of basic genetic algorithm cannot solve the problem of large amount of calculation very well, it is easy to fall into "premature". So this paper use an improved genetic algorithm, the hybrid genetic algorithm to search the optimal value of $Q$ and $R$.

The weight matrixes are generally used in the form of a diagonal matrix for the double inverted pendulum system. Parameters $Q$ and $R$ can be optimized by using the following representation

$$
Q=\operatorname{diag}\left(q_{1}, q_{2}, q_{3}, q_{4}, q_{5}, q_{6}\right), R=q_{7}
$$

Thus objective fitness function ${ }^{[8]}$ can be simplified as:

$$
f(Q, R)=1 /\left(q_{1} x^{2}{ }_{1}+q_{2} x^{2}{ }_{2}+q_{3} x^{2}{ }_{3}+q_{4} x^{2}{ }_{4}+q_{5} x^{2}{ }_{5}+q_{6} x^{2}{ }_{6}+\mathrm{q}_{7} u^{2}\right)
$$

The concrete steps of optimization of hybrid genetic algorithm are as follows:

(1) Coding and set the parameters. This paper chooses the real number coding;

(2) Initialization of population;

(3) Calculation of group on each individual fitness function value;

(4) According to individual fitness value by a rule determined choice into the next generation of the individual;

(5) Cross operation on population;

(6) Mutate operation on population;

(7) Run fmincon optimization algorithms for the individual in the population;

(8) If it don't meet certain conditions, then transferred to step (3) , otherwise go to (9);

(9) The output value of the fitness function in population is the satisfactory solution of the problem or the optimal solution.

Hybrid Genetic Algorithm flow chart are shown below.

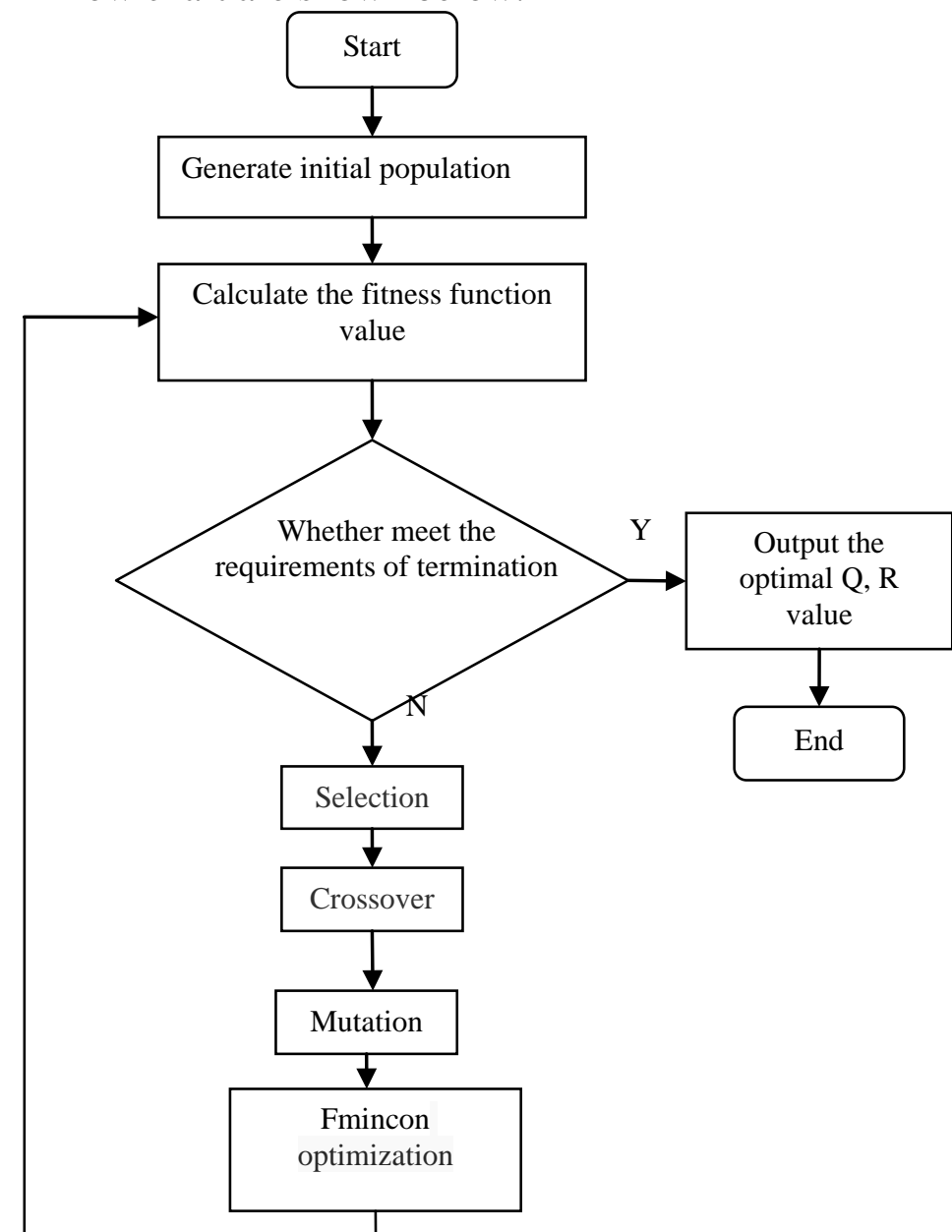

Fig .2: Flowchart of hybrid genetic algorithm 


\section{Experiments and results analysis}

Simulink simulation. In the simulation process, the paper uses real-coded method. The parameters to be optimized are shown in formula (9). Matrix $Q$ values range from 0 to 1000, $R$ values range from 0 to 5 . The initial population number is 80 . The crossover probability is 0.8 , and mutation probability is 0.01 . It is concluded that the value of the objective function $\mathrm{f}$ with algebraic evolutionary process are shown in figure 3 .

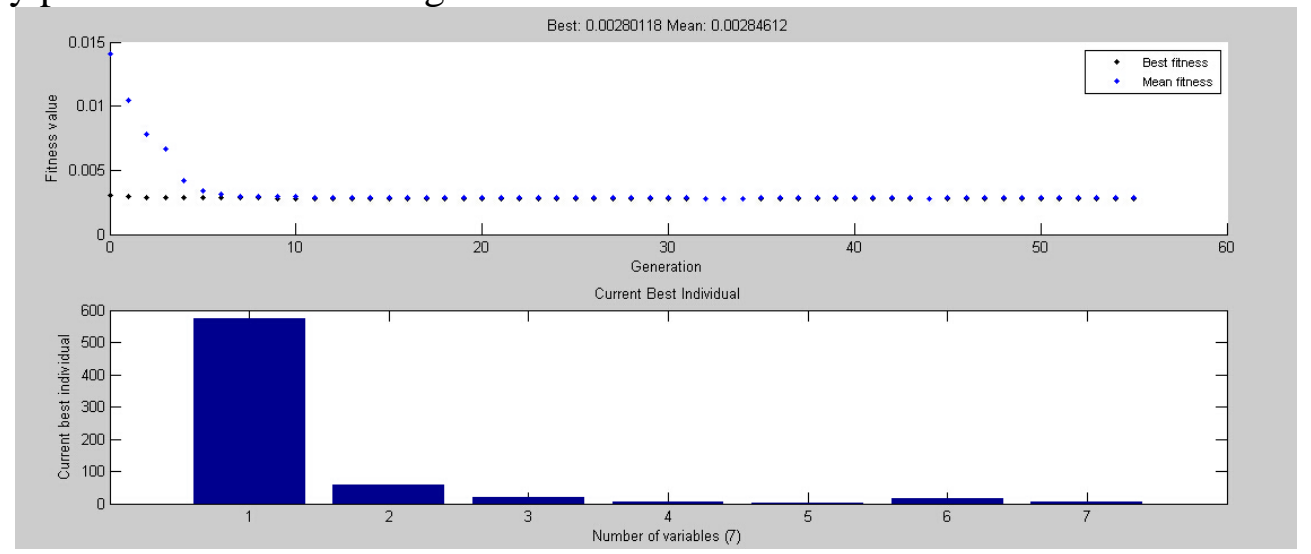

Fig .3: Matlab Hybrid Genetic Algorithm value

After the 55 generation search to obtain a satisfactory solution, the result is:

$$
Q=\operatorname{diag}(575.30,57.17,19.16,5.30,1.27,14.59), R=4.99
$$

Feedback control matrix can be obtained through the Matlab command $K=\operatorname{lqr}(A, B, Q, R)$ :

$$
K=[10.72,90.36,-138.48,11.31,3.34,-22.46]
$$

Using the above parameters optimized in Simulink simulation environment running situation can be seen as follows:

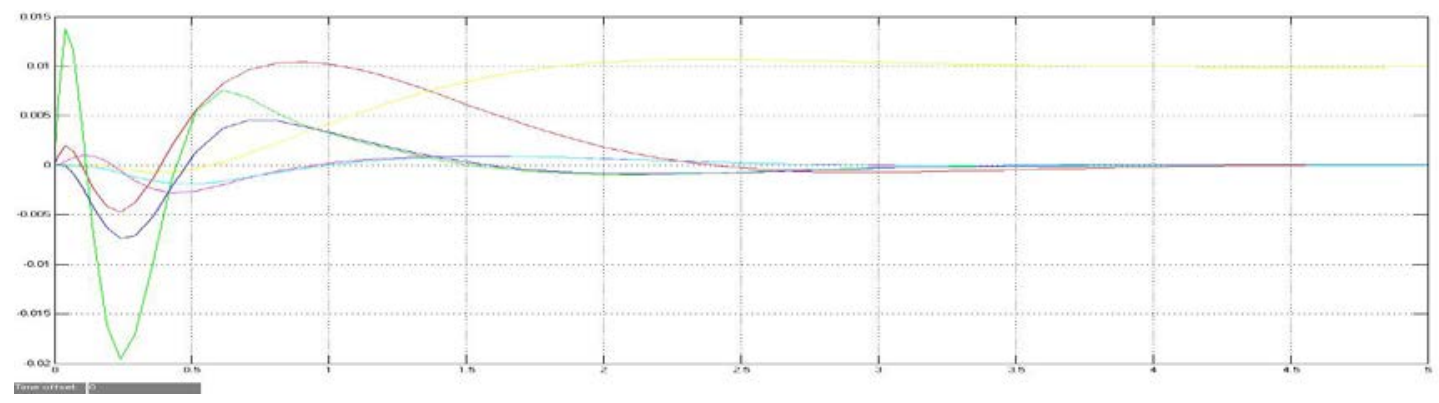

Fig. 4: Inverted pendulum simulation curve

The analysis above shows that, using the optimal controller of the hybrid genetic algorithm can make the car reached the stable point in the vicinity of 2.5S. The short adjustment time and small overshoot meet the design requirements.

Physical control. Googol inverted pendulum control system includes computers, motion control card, servo system, the inverted pendulum body, optical encoder and feedback measurement of several major components. It is a closed loop interdependent system. Through the internal control algorithm motion control card get a corresponding control value to achieve this control decisions.

The feedback matrix $\mathrm{K}$ is used for the actual double inverted pendulum system experiment control device, its control effect is as shown in fig 5.

From top to bottom the figures are real-time car position and the real-time angle of pendulum one and two. It can be seen, the car swings in a place after the inverted pendulum system stability, and it tends to be stable. Both pendulums are in the upright state. It has better control effect and reached the requirements for the control of the system. The final results show that: the controller has well robustness and stability. 
$\mathrm{x}$

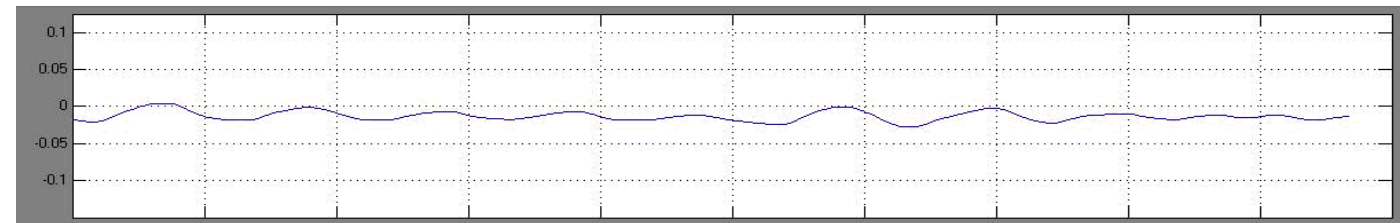

$\theta_{1}$

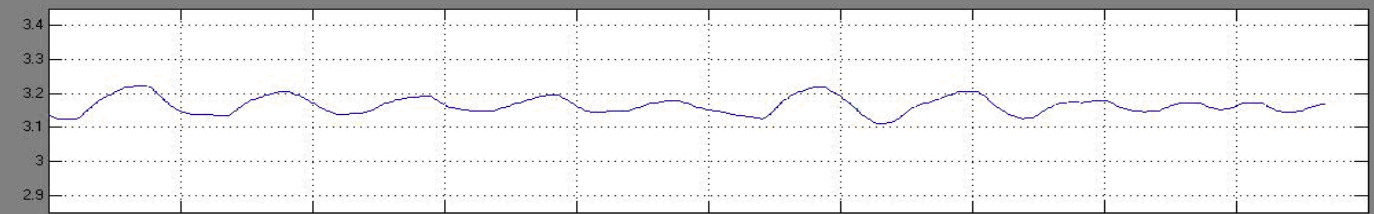

$\theta_{2}$

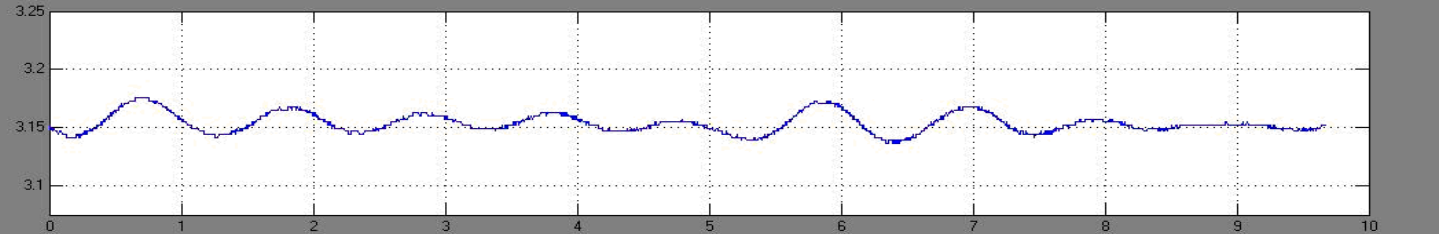

Fig .5: Straight handstand double real-time control results

Applying a disturbance to the system in time $t=3 \mathrm{~s}$ during the process of the inverted pendulum system and stable operation. The results of real-time response are shown in fig 6 .

$\mathrm{X}$

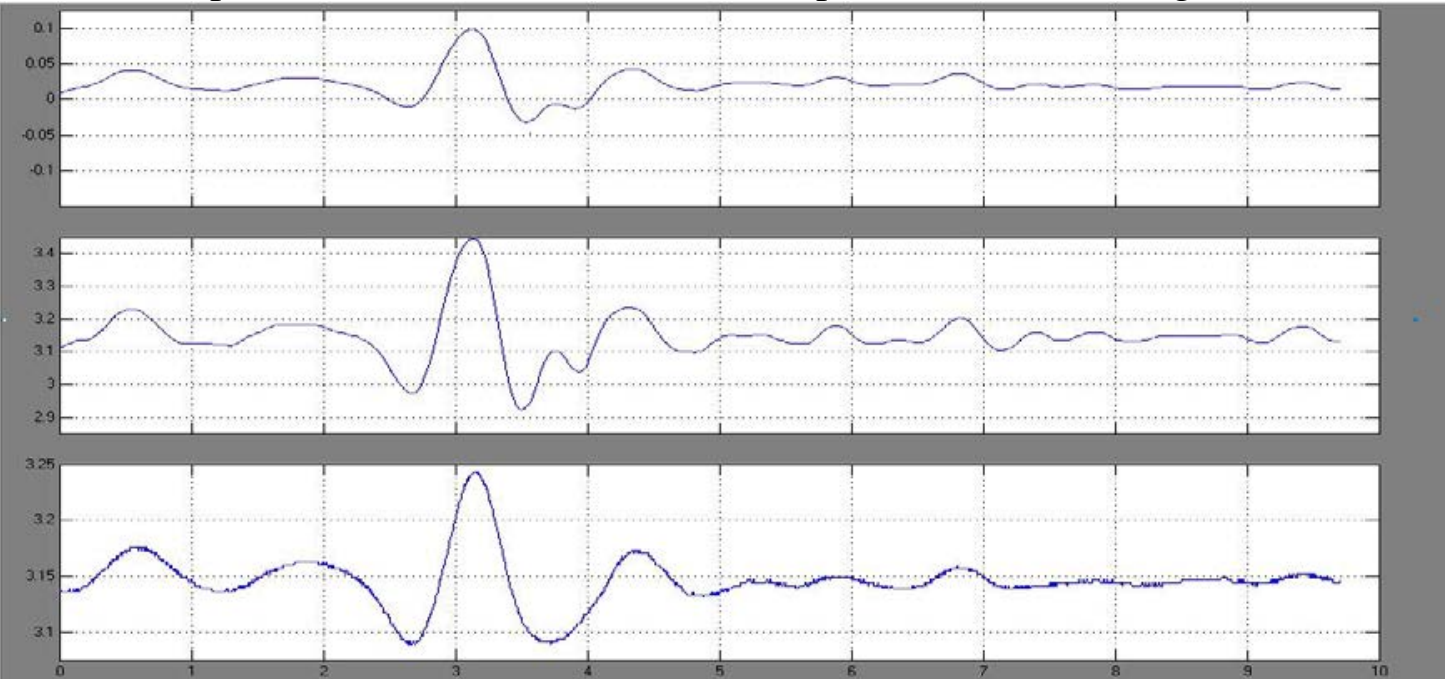

Fig. 6: Double inverted pendulum LQR controller interference curve

From the figure, we can see that the inverted pendulum system can get an excellent control condition when using hybrid genetic algorithm to get the matrix $\mathrm{K}$, with strong anti-interference ability.

\section{Conclusions}

Aiming at the characteristics of difficulty to determine the weighted matrix $\mathrm{Q}$ and $\mathrm{R}$ in $\mathrm{LQR}$, this paper proposed a kind of LQR controller design method based on genetic algorithm. The selection of the weighting matrix is expressed as a multi-objective optimization problem, and chosen the genetic algorithm to optimize weighted matrix parameters. But there are other issues precocious in basic genetic algorithm, so a hybrid genetic algorithm of LQR controller design is proposed. First using the genetic algorithm for global optimization, the parameters in the LQR through its automatic search function to determine the approximate optimal value of the weighted matrix $Q$ and $\mathrm{R}$, and then uses the fmincon function to local optimization value to determine the ultimate value of $\mathrm{Q}$ and $\mathrm{R}$. In the simulation and the actual testing process, this hybrid genetic algorithm has small overshoot and strong anti-interference. The control effect is good. This algorithm can avoid heavy and complicated work, improve the work efficiency, and has strong practicability. In this paper, the idea of hybrid genetic algorithm can be applied in multistage inverted pendulum parameters for master complex function optimization. 


\section{Acknowledgements}

This paper is supported by Institute of electrical engineering, Hebei University of Science and Technology and Institute of Applied Mathematics, Hebei Academy of Sciences.

\section{Corresponding author}

Chaoying Liu, research areas: study on the application of intelligent control, E-mail: liucy@hebust.edu.cn.

\section{References}

[1] Linsen Zeng, The robust LQR optimal control of inverted pendulum. Sichuan, Southwest Jiao Tong University,2014

[2] Zheying Song, Xueling Song, Chaoying Liu, Yingbao Zhao. Research on Real-time Simulation and Control of Linear 1-stage Inverted Pendulum, Journal of Computers, 2013, 8(4) ,896-903

[3] Low KS, Wong T S. Optimization of a Hard Drive Servo System Using Multiobjective Genetic Algorithm. Proceedings of the 2004 IEEE Conference on Cybernetics and Intelligent Systems, Singapore, 2004. 705-710

[4] Zhimin Yang, Yanhai Zheng, Xianyi Zhuang. A review of the application of genetic algorithm in control field, Information and Control ,2000,29 (4): 329-335

[5] Qing Wang, Yingxin Zhang. the optimal controller design of the inverted pendulum experiment system based on genetic algorithm.Laboratory Research and Exploration, 2010, 29 (5): $22-25$

[6] Haiyun Chen,Zhenhua Du, Ningbo Zou,Shiming Jiang. Design of LQR controller for an inverted pendulum multi-population genetic algorithm. Control Engineering, 2014, 21 (3): 391-394

[7] Googol Technology (Shenzhen) Co., Ltd., Inverted pendulum experimental principle and automatic control v2.0. Shenzhen: Googol Technology Co., Ltd., 2005

[8] Yingjie Lei. Genetic algorithm toolbox and its application in MATLAB, Xi'an university of electronic science and technology press, 2005

[9] Qing Jie,Xiaoqiang Zhao. Genetic Algorithm Coding Strategies, Gansu Science and Technology,2013,29(2):13-16

[10] Yu Chen.Three kinds of improved methods of genetic algorithm and research, Computer CD Software and Applications,2014(7),116-118 